\title{
Sequence variation in the Toxoplasma gondii $e I F 4 A$ gene among strains from different hosts and geographical locations
}

\author{
J. Chen ${ }^{1 *}$, S.F. Fang ${ }^{2 *}$, D.H. Zhou ${ }^{1}$, Z.Y. Li ${ }^{1}$, G.H. Liu ${ }^{1,3}$ and X.Q. Zhu ${ }^{1,3}$ \\ ${ }^{1}$ State Key Laboratory of Veterinary Etiological Biology, \\ Key Laboratory of Veterinary Parasitology of Gansu Province, \\ Lanzhou Veterinary Research Institute, Chinese Academy of Agricultural Sciences, \\ Lanzhou, Gansu Province, China \\ ${ }^{2}$ College of Animal Science and Technology, Hebei North University, \\ Zhang Jiakou, Hebei Province, China \\ ${ }^{3}$ College of Veterinary Medicine, Hunan Agricultural University, \\ Changsha, Hunan Province, China \\ *These authors contributed equally to this study. \\ Corresponding authors: D.H. Zhou / X.Q. Zhu \\ E-mail: donghui822002@163.com / xingquanzhu1@hotmail.com
}

Genet. Mol. Res. 13 (2): 3356-3361 (2014)

Received January 25, 2013

Accepted June 14, 2013

Published April 29, 2014

DOI http://dx.doi.org/10.4238/2014.April.29.14

\begin{abstract}
Toxoplasma gondii is an opportunistic protozoan parasite that infects a wide range of animals, including humans. The T. gondii eukaryotic translation initiation factor 4A (eIF4A) protein is expressed in the tachyzoite, but its expression is markedly downregulated in the bradyzoite, and it is therefore considered to be associated with tachyzoite virulence. The present study examined sequence variation in the eIF4A gene among nine strains of different genotypes from different hosts and geographical localities using polymerase chain reaction amplification, sequence analysis, and phylogenetic reconstruction by Bayesian inference. The complete
\end{abstract}


genomic sequence of the eIF4A gene was $3156 \mathrm{bp}$ in length in the strain $\mathrm{TgCgCaI}, 3153 \mathrm{bp}$ in the strain MAS, $3152 \mathrm{bp}$ in the strain TgPNY, and $3154 \mathrm{bp}$ in the other six strains. Sequence analysis identified 29 $(0-0.8 \%)$ variable nucleotide positions among all strains, with 16 of these variations located in the coding region, while the other 12 were distributed between the two introns. Phylogenetic analyses revealed that these eIF4A sequences were not effective molecular markers for intra-species phylogenetic analysis and differential identification of $T$. gondii strains from different hosts and geographical locations. This study demonstrated the existence of low sequence variation in the eIF4A gene, suggesting that $T$. gondii eIF4A may represent a suitable candidate vaccine against toxoplasmosis.

Key words: Toxoplasma gondii; Toxoplasmosis; Sequence variation; Eukaryotic translation initiation factor 4A protein; Phylogenetic analysis

\section{INTRODUCTION}

Toxoplasma gondii is an important zoonotic protozoan with a global distribution infecting virtually all warm-blooded animals, including humans (Montoya and Liesenfeld, 2004; Nardoni et al., 2011; Zhou et al., 2011; Chen et al., 2012; Tian et al., 2012). Normally, infections in humans are asymptomatic or sub-clinical, although they can cause severe diseases in immunocompromised individuals, such as congenitally infected individuals, AIDS patients, and those with tumors or recovering from transplantation operations (Weiss and Dubey, 2009). Additionally, toxoplasmosis can lead to abortion or congenital toxoplasmosis in all types of livestock, especially in sheep and goats (Innes, 2010), and thus cause considerable economic losses to the livestock industry (Fayer et al., 2004; Dubey et al., 2005).

The eukaryotic translation initiation factor eIF4A can control cell proliferation and morphogenesis, which is critical for parasite growth and survival (Tuteja and Pradhan, 2009, 2010). Moreover, the T. gondii eIF4A protein is expressed in the tachyzoite, but is markedly downregulated in the bradyzoite, and is therefore considered to be associated with tachyzoite virulence (Gastens and Fischer, 2002). However, little is known regarding sequence variation of the eIF4A gene among different $T$. gondii strains, despite its important biological impacts. Therefore, the objective of this study was to examine sequence diversity in the eIF4A gene among $T$. gondii strains from different hosts and geographical regions.

\section{MATERIAL AND METHODS}

\section{T. gondii isolates}

A total of nine $T$. gondii strains originating from different hosts and geographic locations were used for analysis in this study (Table 1). These $T$. gondii isolates were genotyped and genomic DNA (gDNA) was prepared as described previously (Zhou et al., 2009; Su et al., 2010; Zhou et al., 2010). 
Table 1. Details of Toxoplasma gondii strains used in the present study.

\begin{tabular}{llll}
\hline Strain & Host & Geographical origin & Genotype* \\
\hline GT1 & Goat & United States & Reference, Type I, ToxoDB \#10 \\
RH & Human & France & Reference, Type I, ToxoDB \#10 \\
PTG & Sheep & United States & Reference, Type II, ToxoDB \#1 \\
CTG & Cat & United States & Reference, Type III, ToxoDB \#2 \\
TgCgCa1 & Cougar & Canada & Reference, ToxoDB \#66 \\
MAS & Human & France & Reference, ToxoDB \#17 \\
TgPNY & Pig & Luying, Henan, China & Type I, ToxoDB \#10 \\
Prugniaud (PRU) & Human & France & Type II, ToxoDB \#1 \\
QHO & Sheep & Huzhu, Qinghai, China & Type II, ToxoDB \#1 \\
\hline
\end{tabular}

*Based on genotyping results of Zhou et al. (2009, 2010), Su et al. (2010).

\section{Polymerase chain reaction (PCR) amplification}

To obtain amplicons of the long genomic sequence of the eIF4A gene more easily, the entire sequence of the gene was separated into two amplified fragments by PCR amplification of the gDNA of individual strains using two pairs of oligonucleotide primers: CJ-eIF4A-1A (forward primer: 5'-AGAAACTGATGCTGCTATA-3') and CJ-eIF4A-1B (reverse primer: 5'-TCTGAGCATCTTACTGGTG-3') were used for the amplification of the first fragment; CJ-eIF4A-2A (forward primer: 5'-AGAAACTGATGCTGCTATA-3') and CJ-eIF4A-2B (reverse primer: 5'-TCTGAGCATCTTACTGGTG-3') were used for the amplification of the second fragment, and $100 \mathrm{bp}$ overlaps between the $5^{\prime}$ termination of the first fragment and the 3 ' termination of the second fragment of the genomic sequence of the eIF4A gene were designed for sequence splicing. The amplification reaction was carried out in a 25 $\mu \mathrm{L}$ volume, containing $10 \mathrm{mM}$ Tris- $\mathrm{HCl}, \mathrm{pH} 8.4,50 \mathrm{mM} \mathrm{KCl}, 3 \mathrm{mM} \mathrm{MgCl}, 250 \mu \mathrm{M}$ each dNTP, $0.2 \mu \mathrm{M}$ of each primer, 100-200 ng template DNA, and 0.25 U La Taq polymerase (TaKaRa). Amplification of DNA samples from individual strains, which were carried out in a thermocycler (Biometra), were denatured at $94^{\circ} \mathrm{C}$ for $10 \mathrm{~min}$ (initial denaturation), followed by 35 cycles consisting of $94^{\circ} \mathrm{C}$ for $30 \mathrm{~s}$ (denaturation), $58.1^{\circ} \mathrm{C}$ for $30 \mathrm{~s}$ (annealing for the first fragment) or $56.6^{\circ} \mathrm{C}$ for $30 \mathrm{~s}$ (annealing for the second fragment), $72^{\circ} \mathrm{C}$ for 2 min (extension), and a final extension step at $72^{\circ} \mathrm{C}$ for $10 \mathrm{~min}$. Confirmation of the PCR amplifications was carried out by electrophoresis on $1 \%(\mathrm{w} / \mathrm{v})$ agarose gel, stained with GoldenView ${ }^{\mathrm{TM}}$, and photographed using a gel documentation system (UVP GelDoc-It ${ }^{\mathrm{TM}}$ Imaging System, Cambridge, UK).

\section{Sequencing of the eIF4A amplicons}

To ensure the accuracy and integrity of eIF4A sequences from individual strains, the eIF4A PCR products were purified using the spin columns according to manufacturer recommendations (Wizard ${ }^{\mathrm{TM}}$ PCR-Preps DNA Purification System, Promega, USA), 1igated with the pMD 18-T vector (TaKaRa), and then transformed into JM109 competent cells (Promega). Following the screening by PCR amplification and enzymatic digestion, the positive colonies were sequenced by the Shanghai Songon Biological Engineering Biotechnology Company on an ABI377 automated DNA sequencer (BigDye Terminator Chemistry). 


\section{Sequence analysis and phylogenetic reconstruction}

The eIF4A gene sequences obtained from different $T$. gondii strains were aligned using the multiple sequence alignment program ClustalX 1.83 (Thompson et al., 1997), and sequence variation was determined among the examined $T$. gondii strains. Phylogenetic reconstructions based on the complete eIF4A gene sequences of different $T$. gondii strains were performed using Bayesian inference (BI). BI analyses were conducted with four independent Markov chains run for 10,000,000 metropolis-coupled MCMC generations, sampling a tree every 10,000 generations in MrBayes 3.1.1 (Ronquist and Huelsenbeck, 2003). The first 250 trees were omitted as burn-ins, and the remaining trees were used to calculate Bayesian posterior probabilities (PP).

\section{RESULTS AND DISCUSSION}

The PCR product of the eIF4A gene appeared to be approximately $3000 \mathrm{bp}$ in length on the agarose gel in all nine $T$. gondii strains, and the amplicons of all strains were sequenced subsequently. The entire genomic sequence of the eIF4A gene was $3156 \mathrm{bp}$ in length for the strain $\mathrm{TgCgCaI}, 3153 \mathrm{bp}$ for the strain MAS, and $3152 \mathrm{bp}$ for the strain TgPNY, and $3154 \mathrm{bp}$ for the other six strains. Analysis of all the nine eIF4A genomic and cDNA sequences revealed two introns in the eIF4A gene (Table 2). There were 35 transitions $(\mathrm{C}<->\mathrm{T}, \mathrm{T}<->\mathrm{G}, \mathrm{A}<->\mathrm{C}$, and $\mathrm{A}<->\mathrm{G})$ and 6 transversions $(\mathrm{A}<->\mathrm{T}$ and $\mathrm{C}<->\mathrm{G})$ in Tg-eIF4A genomic DNA sequences, 14 transitions $(\mathrm{A}<->\mathrm{G}$ and $\mathrm{C}<->\mathrm{T})$ and 3 transversions $(\mathrm{A}<->\mathrm{T}$ and $\mathrm{C}<->\mathrm{G})$ in Tg-eIF4A coding regions, and 11 transitions $(\mathrm{A}<->\mathrm{G}$ and $\mathrm{C}<->\mathrm{T})$ and 2 transversions $(\mathrm{A}<->\mathrm{T}$ and $\mathrm{C}<->\mathrm{G})$ in intron regions (Table 2). The alignment of all nine sequences revealed a total of $29(0-0.8 \%)$ variable nucleotide positions among all strains, 16 of which were in the coding region of the gene and the other 12 were distributed between the two introns, resulting in a low genetic diversity, which was similar to our previous reports of MIC13 (Ren et al., 2012), PLP1 (Yan et al., 2011), and other genes in clonal lineages of $T$. gondii (Khan et al., 2009).

Table 2. Characteristics of Toxoplasma gondii eIF4A (Tg-eIF4A) gene sequences including coding regions and
introns among different strains.
\begin{tabular}{lcccc}
\hline Isolates & Tg-eIF4A DNA & Tg-eIF4A cDNA & First & Second \\
\cline { 3 - 5 } & & & $554-556$ & 242 \\
\hline Length (bp) & $3152-3156$ & 1239 & $45.29-45.90$ & $47.83-48.74$ \\
$\mathrm{~T}+\mathrm{A}(\%)$ & $46.86-47.07$ & $45.78-46.06$ & 9 & 2 \\
Transition & 35 & 14 & 2 & 0 \\
Transversion & 6 & 3 & 4.5 & - \\
$\mathrm{R}$ & 5.8 & 0.7 & 0.6 & $0-0.4$ \\
Distance (\%) & $0-0.8$ & $0-0.8$ & & \\
\hline
\end{tabular}

$\mathrm{R}=$ transition/transversion.

Phylogenetic reconstruction of all nine $T$. gondii strains based on eIF4A sequence data revealed that the eIF4A gene sequences could not readily differentiate $T$. gondii strains belonging to different genotypes (Figure 1), suggesting that the eIF4A DNA sequences were not an ideal genetic marker for intra-species phylogenetic analysis or differentiation of the examined $T$. gondii strains into their respective genotypes. However, the low sequence varia- 
tion $(0-0.8 \%)$ in the eIF4A gene suggests that eIF4A may represent a good candidate vaccine against $T$. gondii infection, and this has been supported by our recent study (Chen et al., 2013).

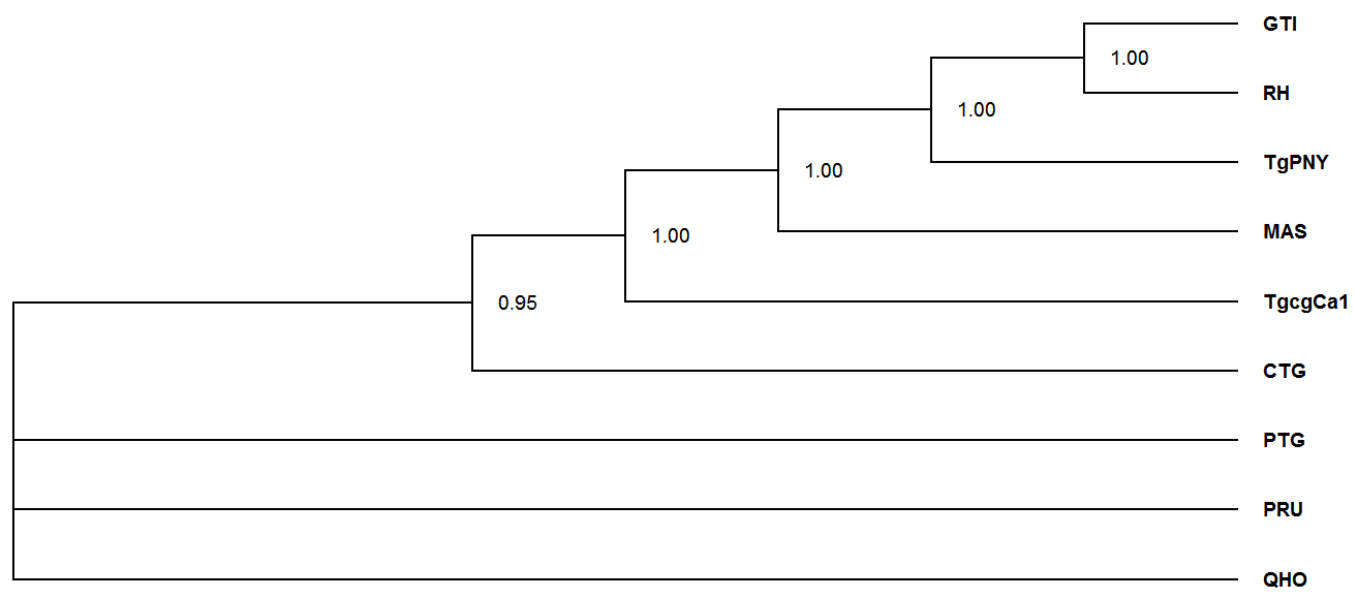

Figure 1. Phylogram of nine Toxoplasma gondii strains determined by analysis of the entire sequences of the eIF4A genomic region. The tree was built by Bayesian inference (BI) analysis. The numbers along branches indicate bootstrap values resulting from BI analyses.

In conclusion, the present study demonstrated low sequence variability in the eIF4A locus among $T$. gondii strains of different genotypes from different hosts and geographical regions, indicating that eIF4A is not a suitable marker for studying genetic variation or the population genetic structure of $T$. gondii isolates.

\section{ACKNOWLEDGMENTS}

Research partially supported by the National Natural Science Foundation of China (Grant \#31228022, \#31230073, \#31172316, and \#31101812), the International Science and Technology Cooperation Project of Gansu Province (Grant \#1204WCGA023), and The Science Fund for Creative Research Groups of Gansu Province (Grant \#1210RJIA006).

\section{Conflicts of interest}

The authors declare no conflict of interest.

\section{REFERENCES}

Chen J, Xu MJ, Zhou DH, Song HQ, et al. (2012). Canine and feline parasitic zoonoses in China. Parasit. Vectors 5: 152. Chen J, Huang SY, Li ZY, Yuan ZG, et al. (2013). Protective immunity induced by a DNA vaccine expressing eIF4A of Toxoplasma gondii against acute toxoplasmosis in mice. Vaccine 31: 1734-1739.

Dubey JP, Hill DE, Jones JL, Hightower AW, et al. (2005). Prevalence of viable Toxoplasma gondii in beef, chicken, and pork from retail meat stores in the United States: risk assessment to consumers. J. Parasitol. 91: 1082-1093.

Fayer R, Dubey JP and Lindsay DS (2004). Zoonotic protozoa: from land to sea. Trends Parasitol. 20: 531-536.

Gastens MH and Fischer HG (2002). Toxoplasma gondii eukaryotic translation initiation factor 4A associated with 
tachyzoite virulence is downregulated in the bradyzoite stage. Int. J. Parasitol. 32: 1225-1234.

Innes EA (2010). Vaccination against Toxoplasma gondii: an increasing priority for collaborative research? Expert Rev. Vaccines 9: 1117-1119.

Khan A, Taylor S, Ajioka JW, Rosenthal BM, et al. (2009). Selection at a single locus leads to widespread expansion of Toxoplasma gondii lineages that are virulent in mice. PLoS Genet. 5: e1000404.

Montoya JG and Liesenfeld O (2004). Toxoplasmosis. Lancet 363: 1965-1976.

Nardoni S, Angelici MC, Mugnaini L and Mancianti F (2011). Prevalence of Toxoplasma gondii infection in Myocastor coypus in a protected Italian wetland. Parasit. Vectors 4: 240.

Ren D, Zhou DH, Xu MJ, Zhou Y, et al. (2012). Sequence variation in Toxoplasma gondii MIC13 gene among isolates from different hosts and geographical locations. Afr. J. Microbiol. Res. 6: 1333-1337.

Ronquist F and Huelsenbeck JP (2003). MrBayes 3: Bayesian phylogenetic inference under mixed models. Bioinformatics 19: $1572-1574$.

Su C, Shwab EK, Zhou P, Zhu XQ, et al. (2010). Moving towards an integrated approach to molecular detection and identification of Toxoplasma gondii. Parasitology 137: 1-11.

Thompson JD, Gibson TJ, Plewniak F, Jeanmougin F, et al. (1997). The CLUSTAL_X windows interface: flexible strategies for multiple sequence alignment aided by quality analysis tools. Nucleic Acids Res. 25: 4876-4882.

Tian YM, Dai FY, Huang SY, Deng ZH, et al. (2012). First report of Toxoplasma gondii seroprevalence in peafowls in Yunnan Province, Southwestern China. Parasit. Vectors 5: 205.

Tuteja R and Pradhan A (2009). Isolation and functional characterization of eIF4F components and poly(A)-binding protein from Plasmodium falciparum. Parasitol. Int. 58: 481-485.

Tuteja R and Pradhan A (2010). PfeIF4E and PfeIF4A colocalize and their double-stranded RNA inhibits Plasmodium falciparum proliferation. Commun. Integr. Biol. 3: 611-613.

Weiss LM and Dubey JP (2009). Toxoplasmosis: A history of clinical observations. Int. J. Parasitol. 39: 895-901.

Yan HK, Song HQ, Zhou Y, Ren D, et al. (2011). Sequence variation in perforin-Like protein 1 gene among six Toxoplasma gondii strains. J. Anim. Vet. Adv. 10: 2244-2247.

Zhou P, Zhang H, Lin RQ, Zhang DL, et al. (2009). Genetic characterization of Toxoplasma gondii isolates from China. Parasitol. Int. 58: 193-195.

Zhou P, Nie H, Zhang LX, Wang HY, et al. (2010). Genetic characterization of Toxoplasma gondii isolates from pigs in China. J. Parasitol. 96: 1027-1029.

Zhou P, Chen Z, Li HL, Zheng H, et al. (2011). Toxoplasma gondii infection in humans in China. Parasit. Vectors 4: 165. 